\title{
SUMS OF $k$ TH POWERS IN THE RING OF POLYNOMIALS WITH INTEGER COEFFICIENTS
}

\author{
BY TED CHINBURG AND MELVIN HENRIKSEN ${ }^{1}$ \\ Communicated by Robert Fossum, August 1, 1974
}

Suppose $R$ is a ring with identity element and $k$ is a positive integer. Let $J(k, R)$ denote the subring of $R$ generated by its $k$ th powers. If $Z$ denotes the ring of integers, then $G(k, R)=\{a \in Z: a R \subset J(k, R)\}$ is an ideal of $Z$.

Let $Z[x]$ denote the ring of polynomials over $Z$ and suppose $a \in R$. Since the map $p(x) \rightarrow p(a)$ is a homomorphism of $Z[x]$ into $R$, the wellknown identity (see [3, p. 325])

$$
k ! x=\sum_{i=0}^{k-1}(-1)^{k-1-i}\left(\begin{array}{c}
k-1 \\
i
\end{array}\right)\left\{(x+i)^{k}-i^{k}\right\}
$$

in $Z[x]$ tells us that $k ! \in G(k, Z[x]) \subseteq G(k, R)$. Since $Z$ is a cyclic group under addition, this shows that $G(k, R)$ is generated by its minimal positive element, which we denote by $m(k, R)$. Abbreviating $m(k, Z[x])$ by $m(k)$, we then have $m(k, R) \mid m(k)$ and $m(k) \mid k !$.

Thus $m(k)$ is the smallest positive integer $a$ for which there is an identity of the form

$$
a x=\sum_{i=1}^{n} a_{i}\left[g_{i}(x)\right]^{k}
$$

where $a_{1}, \cdots, a_{n} \in Z$ and $g_{1}(x), \cdots, g_{n}(x) \in Z[x]$.

On differentiating (2) with respect to $x$ we have $k \mid m(k)$. Thus if $R$ is any ring with identity,

$$
k|m(k), \quad m(k, R)| m(k), \quad \text { and } m(k) \mid k ! \text {. }
$$

For any $k \geqslant 1$ in $Z$, let $P_{1}(k)$ denote the set of primes less than $k$ that divide $k$, and let $P_{2}(k)$ denote the set of primes less than $k$ that fail to divide $k$. If $p$ is a prime and $r \geqslant 1, m>1$ are integers, then a number

$A M S$ (MOS) subject classifications (1970). Primary 10M05, 10B25, 12C15; Secondary 13 F20.

$1_{W e}$ are indebted to $H$. Edgar and W. LeVeque for valuable references. 
of the form $\left(p^{m r}-1\right) /\left(p^{r}-1\right)$ is called a p-power sum. We adopt the convention that the product of an empty set of integers is 1 . The main theorem of this paper is the following.

THEOREM 1. If $k$ is a positive integer then

$$
m(k)=k \Pi\left\{p^{\alpha_{k}(p)}: p \in P_{1}(k)\right\} \Pi\left\{p^{\beta_{k}(p)}: p \in P_{2}(k)\right\}
$$

where

$$
\begin{aligned}
& \alpha_{k}(p)=1 \quad \text { if } p \text { is odd. } \\
& \alpha_{k}(2)= \begin{cases}2 & \text { if }\left(2^{j}-1\right) \mid k \text { for some } j \geqslant 2, \\
1 & \text { otherwise. }\end{cases} \\
& \beta_{k}(p)= \begin{cases}1 & \text { if some p-power-sum divides } k, \\
0 & \text { otherwise. }\end{cases}
\end{aligned}
$$

A proof of this theorem will appear in [2]. Appropriate identities are developed in various homomorphic images of $Z[x]$ and lifted. Except for (b), these homomorphic images are Galois fields. A constructive but impractical algorithm is developed for obtaining identities of the form (2) with $a=$ $m(k)$. The reader may easily verify the entries in the following table of values of $m(k) / k$ for $1 \leqslant k \leqslant 20$.

\begin{tabular}{c|c|c|c|c|c|c|c}
$k$ & 1 & 2 & 3 & 4 & 5 & 6 & 7 \\
\hline$m(k) / k$ & 1 & 1 & 2 & $2 \cdot 3=6$ & 2 & $4 \cdot 3 \cdot 5=60$ & 2
\end{tabular}

\begin{tabular}{c|c|c|c|c|c}
$k$ & 8 & 9 & 10 & 11 & 12 \\
\hline$m(k) / k$ & $2 \cdot 3 \cdot 7=42$ & $2 \cdot 3=6$ & $2 \cdot 3 \cdot 5=30$ & 1 & $4 \cdot 3 \cdot 5 \cdot 11=660$
\end{tabular}

\begin{tabular}{c|c|c|c|c|c|c}
$k$ & 13 & 14 & 15 & 16 & 17 & 18 \\
\hline$m(k) / k$ & 3 & $4 \cdot 7 \cdot 13=364$ & $2 \cdot 3 \cdot 5=30$ & $2 \cdot 3 \cdot 7=42$ & 2 & $4 \cdot 3 \cdot 5 \cdot 17=1,020$
\end{tabular}

\begin{tabular}{c|c|c|c}
$k$ & 19 & 20 \\
\hline$m(k) / k$ & 1 & $2 \cdot 3 \cdot 5 \cdot 19=570$ &
\end{tabular}

A table of values for $m(k) / k$ for $1 \leqslant k \leqslant 150$ is supplied in [2] together with an algorithm for computing values of $m(k) / k$ efficiently.

If $\Gamma$ is any set of primes, let $S(\Gamma)$ denote the multiplicative semigroup generated by $\Gamma$. Let $T(\Gamma)$ denote the set of $a>1$ in $Z$ for which there is a $d>1$ in $Z$ such that $\left(a^{d}-1\right) /(a-1) \in S(\Gamma)$.

The next theorem yields some information about the distribution of values of $m(k) / k$. Recall that a prime is called a Mersenne (resp. Fermat) prime if $p=2^{n}-1$ (resp. $p=3$ or $p=2^{n}+1$ ) for some integer $n>1$. 
THEOREM 2. Suppose $\Gamma$ is a finite set of primes.

(a) $T(\Gamma)$ is the union of a finite set and $\{a \in Z: a>1$ and $(a+1) \in$ $S(\Gamma)\}$

(b) If $S(\Gamma)$ contains no even integer, then $\{a \in T(\Gamma): a$ is odd $\}$ is finite.

(c) If $2 \notin \Gamma$, then $\{m(k) / k: k \in S(\Gamma)\}$ is bounded. In particular, if $k>1$ is an odd integer, then $\left\{m\left(k^{n}\right) / k^{n}\right\}$ is a bounded sequence.

(d) If $n>1$ is an integer, then $m\left(2^{n}\right) / 2^{n}$ is the product of all the Mersenne primes less than $2^{n}$.

(e) If $p$ is a Fermat prime, then $m\left(p^{n}\right) / p^{n}=2 p$ for every integer $n>1$.

A proof of Theorem 2 is given in [2].

We conclude with some remarks and unsolved problems.

(A) P. Bateman and R. M. Stemmler show in [1, p. 152] that if $\left\{p_{n}\right\}$ is the sequence of primes such that $p_{n}$ is a $q$-power sum for some prime $q$, where $p_{n}$ is repeated if it is a $q$-power sum for more than one prime $q$, then $\Sigma_{n=1}^{\infty} p_{n}^{-1 / 2}<\infty$. Hence such primes are sparsely distributed. Indeed, they state that there are only 814 such primes less than $1.25 \times 10^{10}$, and they exhibit the first 240 of them. In this range $31=\left(2^{6}-1\right) /(2-1)=$ $\left(5^{3}-1\right) /(5-1)$ is the only prime that is a $q$-power sum for more than one prime $q$. For any prime $p, m(p) / p$ is the product of all primes $q$ such that $p$ is a $q$-power sum. It does not seem to be known if there is a positive integer $N$ such that $m(p) / p$ has no more than $N$ prime factors for every prime $p$.

(B) Can the sequence $\left\{m\left(k^{n}\right) / k^{n}\right\}$ be bounded if $k$ is even? By Theorem $2(d),\left\{m\left(2^{n}\right) / 2^{n}\right\}$ is bounded if and only if there are only finitely many Mersenne primes. What if $k$ is even and composite?

(C) By Theorem 2 (c), if $\Gamma$ is a finite set of odd primes, then there is a smallest positive integer $M(\Gamma)$ such that $m(s) / s \leqslant M(\Gamma)$ for every $s \in S(\Gamma)$. By Theorem $2(\mathrm{e}), M(\Gamma)=2 p$ if $\Gamma=\{p\}$ and $p$ is a Fermat prime, and since $(11)^{2}=\left(3^{5}-1\right) /(3-1), M(\{11\}) \geqslant 33$. Is there a general method for computing $M(\Gamma)$ ? What if $|\Gamma|=1$ ?

(D) It is not difficult to prove that if $R$ is a ring with identity for which there is a homomorphism of $R$ onto $Z[x]$, then $m(k, R)=m(k)$. In particular, if $\left\{x_{\alpha}\right\}$ is any collection of indeterminates, then $m\left(k, Z\left[\left\{x_{\alpha}\right\}\right]\right)$ $=m(k)$. 


\section{REFERENCES}

1. P. T. Bateman and R. M. Stemmler, Waring's problem for algebraic number fields and primes of the form $\left(p^{r}-1\right) /\left(p^{d}-1\right)$, Illinois J. Math. 6 (1962), $142-156$. MR 25 \#2059.

2. T. Chinburg and M. Henriksen, Sums of kth powers in the ring of polynomials with integer coefficients, Acta Arith. (submitted).

3. G. H. Hardy and E. M. Wright, The theory of numbers, Oxford Univ. Press, London, 1946.

DEPARTMENT OF MATHEMATICS, HARVEY MUDD COLLEGE, CLAREMONT, CALIFORNIA 91711 